\title{
Parameter-adaption for a vehicle dynamics model for the evaluation of powertrain concept designs
}

\author{
$C$ Angerer ${ }^{1,3, *}, B$ Mößner ${ }^{1}, M$ Lüst $^{1}, S$ Büchner $^{1}, F$ Sträuß1 $^{2,3}$, and $M$ Lienkamp $^{1}$ \\ ${ }^{1}$ Institute of Automotive Technology, Technical University of Munich, Garching, Germany \\ ${ }^{2}$ TUM School of Management, Technical University of Munich, Garching, Germany \\ ${ }^{3}$ Center for Design Research, Stanford University Stanford, CA, USA
}

\begin{abstract}
The powertrain design of multi-motor electric vehicles directly affects not only costs, consumption and acceleration, but also the handling of a vehicle. Therefore, a holistic powertrain design optimization needs to include a vehicle dynamics model in its objective function. While the parameters for the powertrain model result from the design variables that describe the powertrain, the parameters for the vehicle dynamics model must be adapted in a feasible way to ensure comparable results. Therefore, the authors present a method on how to adaptively parametrize a doubletrack vehicle dynamics model for the use in powertrain design optimization. Automated design calculations for all main chassis and suspension parts are used to determine the parameters for the model. A parameter variation proves the plausibility of the approach. The results show that an adaption of the suspension and chassis parameters due to changes in the powertrain make results more comparable but do not compensate for the effects on the vehicle handling. In particular, the steady state longitudinal load distribution still has major influences on the vehicle handling.
\end{abstract}

\section{Introduction}

Design optimization of vehicle powertrains is a state-of-the-art method to find optimal system and component designs within a large solution space. A set of design variables defines a certain design and serves as input for an objective function that represents the system or component behavior. The resulting objectives determine the optimality of the system design and are analyzed by optimization algorithms that are applied to it.

While it is sufficient for internal combustion engine (ICE) vehicles to consider the powertrain and the vehicle's longitudinal dynamics, the design of multi-motor electric vehicle $(\mathrm{EV})$ powertrains can have major influences on the handling of a vehicle because multi-motor electric powertrains have the potential to improve handling and efficiency at the same time [1] (while for ICE the cross-dependencies between efficiency, vehicle dynamics and powertrain design can more easily be separated). As a consequence the vehicle handling must be considered as objective in a powertrain design optimization [2].

\footnotetext{
* Corresponding author: angerer@ftm.mw.tum.de
} 
Therefore the authors develop a powertrain design optimization that includes holistic objectives (costs, consumption, performance and handling). Figure 1 visualizes the overall approach in more detail. A set of design variables (nominal speeds and torques etc.) determines a certain powertrain (topology, choice and sizing of components) [3]. The objective function is split into three main steps. Firstly, the preprocessing calculates parameters for the simulation models such as efficiency maps. Next, driving maneuvers are simulated and thus determine the systems physical behavior. Finally, an evaluation determines the objectives (scalar values) based on the simulation results (physical quantities over time).

The objective function for the design optimization of multi-motor EV also needs to represent the handling properties and must therefore contain a vehicle dynamics model in addition to the powertrain model [2]. Nevertheless, the design variables still only describe the powertrain design and do not provide information about the parameters of the vehicle dynamics model. The parameters cannot be held constant as a variation of the powertrain also causes modifications on chassis and suspension design that must be taken into account to ensure comparable results. Additionally, multi-motor EV powertrains offer more degrees of freedom regarding torque actuation. On the one hand, this is the main reason for its potential regarding efficiency and handling, but on the other hand it requires a torque allocation. This torque allocation must be adaptive to ensure that the different objective values represent the effects of the modified design variables and do not mainly depend on the parameters for the torque allocation.

Therefore, the authors of this paper present an approach for a parameter adaption of a vehicle dynamics model within a powertrain design optimization for multi-motor electric vehicles.

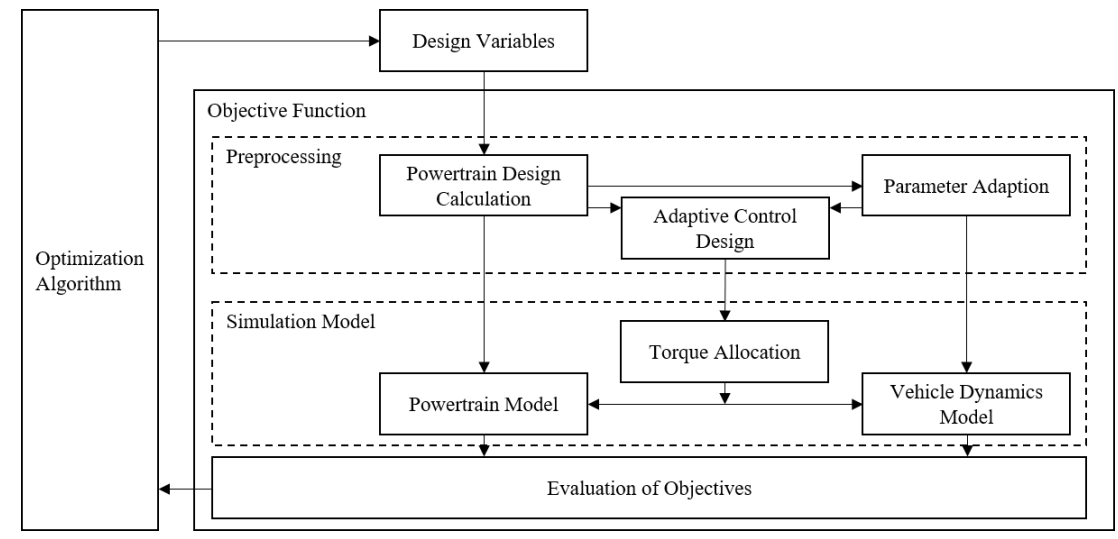

Fig. 1. Optimization Process with Parameter Adaption included in the Objective Function.

\section{Literature Review}

A literature review first proves the relevance of handling aspects in powertrain design optimization. The second subsection reviews examples for vehicle design optimization and the following presents the state of the art in investigations of multi-motor EV. Based on that, the research gap concludes the need for a parameter adaption for vehicle dynamics models to be used in powertrain design optimization.

\subsection{Relevance of Powertrain Design for Vehicle Dynamics}

The powertrain design affects vehicle dynamics mainly with respect to torque and weight distribution. In most cases, ICE vehicles have their motor and gearbox located in the front 
and therefore the weight distribution is determined in advance. The distribution of torque in the longitudinal direction is determined by the powertrain architecture (front-wheel drive (FWD), rear-wheel drive (RWD) or all-wheel drive (AWD)). The lateral distribution of torque usually is equally balanced by common differentials. Torque vectoring systems or limited slip differentials can modify that but are optional components for the vehicle that can be considered separately in addition to any ICE powertrain design.

In contrast to that, electric vehicle powertrains are likely to have more than one motor [4]. This results from the fact that multi-motor electric powertrains can recover more kinetic energy [5] and improve efficiency by more than $2 \%$ [6]. As a consequence, lateral or longitudinal distribution of torque can not only be achieved by equipping the vehicle with extra components such as a torque vectoring unit, but also by the general choice of the powertrain design. This enables a flexible distribution of torque in longitudinal and/or lateral direction that - according to [7] - can influence the handling of a vehicle. It allows for improvements in steady-state as well as transient vehicle characteristics [8] and enhances acceleration on $\mu$-split conditions [6]. Finally, multi-motor electric vehicles even have the potential to improve handling and efficiency at the same time [1]. Therefore, the powertrain design for multi-motor electric vehicles, in particular for electric AWD vehicles, has large effects on the vehicle dynamics.

\subsection{Vehicle Design Optimization}

Design optimization is a state of the art method to find the optimal system design in a large solution space using an objective function, which represents the real system, and considering boundary conditions. Hereby, the solution space defines the ranges of values of the design variables. The objective function represents the correlation between the choice of design variable values and the resulting objective values. An optimization algorithm is applied to find the minimum (or maximum) objective value for any possible set of design variable values within the solution space $[9,10]$. Multi-objective optimization is required when there is more than one objective and an aggregation by using a weighting function is not possible such as for non-convex objective functions [11]. In the following, the authors only consider static optimization (design variables originate from Euclidean Space) in contrast to dynamic optimization or dynamic programming (design variables originate from Hilbert Space) which is used for optimal control problems [9].

Static optimization for vehicle design has various fields of applications. It is applied on component level [12-14] and on system level. On system level, common fields of application are the vehicle structure $[15,16]$, the powertrain design [17-20] as well as the suspension design [21-23]. Each of the approaches for powertrain or suspension design optimization is limited to its field of research and does not take into account further systems such as vehicle dynamics in powertrain design optimization.

\subsection{Adaption of Parameters for Multi-Motor Electric Vehicles Simulation}

Research on multi-motor EVs available in literature [1, 4-6, 24] examines a certain (set of) example(s) but does not optimize the powertrain design holistically. For these applications, parameters of the vehicle dynamics model can be adapted manually or kept constant. In contrast to this, powertrain optimization requires an automated evaluation of the objective function. A manual adaption is not feasible, and the parameters need to be adapted automatically.

\subsection{Research Gap}

The state of the art proved that 
a) powertrain design for multi-motor EV needs to consider vehicle dynamics. Therefore, powertrain design optimization requires a vehicle dynamics model in its objective function to include the vehicle handling in the objectives (Section 2.1.)

b) existing powertrain design optimization approaches in literature do not consider the influences of neighboring systems / other domains (such as vehicle dynamics for powertrain design) (Section 2.2. )

c) existing investigations of multi-motor EV powertrains are limited to exemplary studies of a certain powertrain design or a comparison of a limited number of design options, but do not apply optimization to it (Section 2.3. )

While a) proves the need of considering vehicle dynamics in powertrain design optimization for multi-motor EVs, b) and c) prove that such a holistic powertrain design optimization has not been conducted yet. The integration of a vehicle dynamics model causes the problem that its parameters do not directly result from the design variables as these only describe the powertrain design (Figure 1). Nevertheless, the vehicle dynamics model parameters must be adapted due to changes in weight distribution and total weight, resulting from modifications in the powertrain design.

Therefore, the authors present a method to automatically adapt the model parameters of a double-track vehicle dynamics model (according to [25]) for use in the objective function of a powertrain design optimization approach. The development of the parameter adaption method serves its future application in a holistic powertrain concept optimization for electric vehicles with a focus on multi-motor configurations.

\section{Parameter Adaption for Vehicle Dynamics Model}

According to Figure 1, the design variables serve as inputs for the powertrain design calculations. In the next step, the resulting powertrain parameters are forwarded to the parameter adaption. The final parameters afterwards are forwarded to the vehicle dynamics model, which in this study is an enhanced double-track model according to the implementation by [25]. Figure 2 visualizes the parameter adaption while the following subsections explain each step of the process in detail.

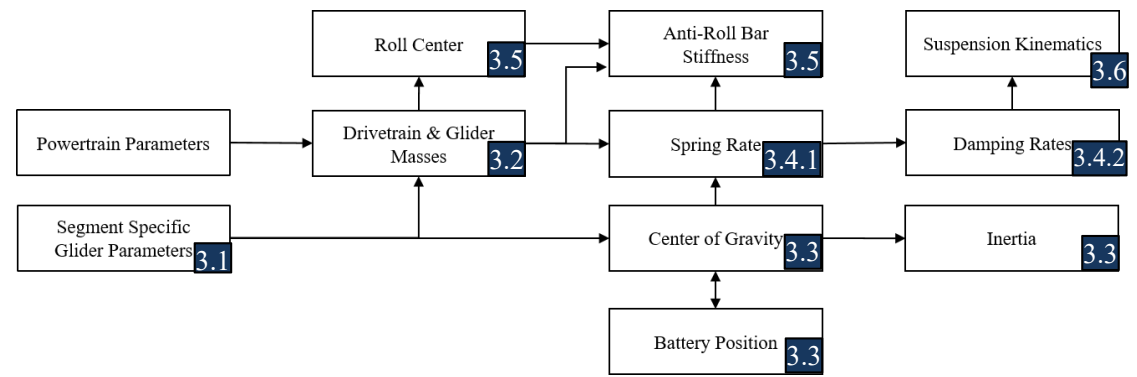

Fig. 2. Visualization of the Parameter Adaption Process.

\subsection{Segment Specific Input Parameters}

The powertrain parameters from powertrain design calculation are input to the parameter adaption and define the powertrain. Furthermore, the glider (vehicle without powertrain) needs to be characterized. Therefore, segment specific glider parameters must be chosen. They can either represent a certain vehicle or a whole vehicle segment using average values. The glider parameters are required as inputs for the subsequent mass calculation of each component. This mass calculation is based on regression analyses by [26]. The segmentspecific glider parameters are: 
- Dimensions: track width, wheel base, height, overhang front and rear,

- Aerodynamic data (drag coefficient, frontal area)

- Geometric data for mass estimation: inclination angles of front screen, side and rear windows

- Tire model, dimensions and rolling resistance

- Comfort and acoustic characteristic values

- Target range for a certain drive cycle

\subsection{Drivetrain \& Glider Masses}

While the powertrain masses result from the powertrain design calculation, the approach in [26] is used to calculate the glider masses. The first step is to estimate the curb weight using regressions based on the vehicle's main dimensions. Then, all component masses are calculated using component-specific regressions. Only the masses of the powertrain remain unchanged, as they have already been determined by the choice of powertrain design. The battery mass is calculated in order to provide the target range at current weight. To accurately represent mass dependent components (such as brakes), this process is repeated iteratively until the error between the newly calculated and the previous weight value is negligibly small. By that, secondary mass effect such as smaller brake dimensions as a result of lighter powertrain are considered.

\subsection{Center of Gravity, Inertia and Battery Position}

Subsequently, the center of gravity of the battery pack is adjusted iteratively in order to achieve an equally balanced longitudinal load distribution. Geometric boundaries [29] resulting from the vehicle package are considered. Therefore, the lateral distance between the battery pack and the vehicles outer end of the side skirts is assumed to be $100 \mathrm{~mm}$ on each side and the battery pack containing the cell stacks to be $100 \mathrm{~mm}$ in height. Taking into consideration the volumetric energy density and a certain desired energy capacity, the required battery length can be deduced. Hereby, battery auxiliaries, such as contactors, battery management systems etc., are assumed to be placed elsewhere above the cell stacks. Knowing the battery length as well as the wheel base, the available clearance for positioning the battery longitudinal direction is determined. Subsequently, the position is adapted within these borders in order to achieve a 50/50 weight distribution.

In the next step, the vehicle's inertia is calculated. By positioning the components and calculating their dimensions, the vehicle's center of gravity as well as the vehicle's inertia can be determined. The results are validated against the inertia estimation method from [27].

\subsection{Suspension Parameters}

The suspension parameters are chosen as a trade-off between vehicle comfort and safety or stability. Therefore, some mechanical quantities have been set constant. They might be adapted for different vehicle types. The values used for further calculations are listed in Table 1.

Table 1. Constant Values for Suspension Design.

\begin{tabular}{|c|c|}
\hline Quantity & Value \\
\hline$\omega_{0}$ & $1,4 \mathrm{~Hz}$ \\
\hline$k_{\omega_{0}}$ & 1.15 \\
\hline$D$ & 0.3 \\
\hline$k_{D}$ & 1.6 \\
\hline$k_{\text {reb,comp }}$ & 2.5 \\
\hline
\end{tabular}




\subsubsection{Spring Rates}

The spring stiffness is designed to achieve a certain undamped Eigen frequency. The total Eigen frequency of the vehicle $\omega_{0}$ in dependence on the vehicles sprung mass $m$ and the spring stiffnesses of front $\mathrm{c}_{\mathrm{f}}$ and rear axles $\mathrm{c}_{\mathrm{r}}$ can be expressed by [28]:

$$
\omega_{0}=\sqrt{\frac{c_{f}+c_{r}}{m}}
$$

By substituting each axle's spring stiffness with respect to their partial mass and their half vehicle Eigen frequency of front $\omega_{f}$ and rear axle $\omega_{r}$

$$
c_{f / r}=\omega_{0, f / r^{2}} \cdot m_{f / r}
$$

and introducing the weight distribution factor $R_{f / r}$

$$
R_{f / r}=\frac{m_{f / r}}{m}
$$

the correlation between the overall vehicle's and the axle's individual Eigen frequencies can be derived:

$$
\omega_{0}=\omega_{0, f^{2}} \cdot R_{f}+\omega_{0, r^{2}} \cdot R_{r}
$$

[28] suggests a certain ratio $k_{\omega_{0}}$ between the half-vehicle Eigen frequencies to achieve good vehicle handling and comfort with respect to pitch dynamics.

$$
k_{\omega_{0}}=\frac{\omega_{0, r}}{\omega_{0, f}}
$$

Using (5) in (4) derives each axle's Eigen frequency. With (2) each axle's spring stiffness can be calculated:

$$
\begin{gathered}
c_{f}=m_{f} \cdot \frac{\omega_{0}}{\sqrt{R_{f}+k_{\omega_{0}}{ }^{2} \cdot R_{r}}} \\
c_{r}=m_{r} \cdot \frac{\omega_{0}}{\sqrt{\frac{1}{k_{\omega}{ }^{2}} \cdot R_{f}+R_{r}}}
\end{gathered}
$$

\subsubsection{Damping Rates}

The damper values are determined in a similar way. The constant target value is the desired Lehr damping coefficient $\mathrm{D}$ for the sprung mass of the full vehicle. It depends on the mass, the spring stiffness and the damping rate of front and rear axle.

$$
D=\frac{d_{f}+d_{r}}{2 \cdot \sqrt{m \cdot\left(c_{f}+c_{r}\right)}}
$$

[28] provides values for $k_{D}$ that describe the ratio between the rear and front axles' Lehr damping coefficients:

$$
k_{D}=\frac{D_{r}}{D_{f}}
$$


Analogous to the spring rates this yields the damping rates of front and rear axles:

$$
\begin{gathered}
d_{f}=2 D \cdot \frac{\sqrt{c_{f}+c_{r}}}{\sqrt{c_{f} \cdot R_{f}}+k_{D} \cdot \sqrt{c_{r} \cdot R_{r}}} \cdot \sqrt{c_{f} \cdot m} \\
d_{r}=2 D \cdot \frac{\sqrt{c_{f}+c_{r}}}{\frac{1}{k_{D}} \cdot \sqrt{c_{f t} \cdot R_{f}}+\sqrt{c_{r} \cdot R_{r}}} \cdot \sqrt{c_{r} \cdot m}
\end{gathered}
$$

In contrast to the springs, dampers for vehicles show a different behavior for different operating directions. The ratio between rebound and compression damping coefficients $k_{\text {reb,comp }}$ is adapted from Preda [30].

$$
k_{r e b, c o m p}=\frac{d_{r e b}}{d_{c o m p}}
$$

Assuming $d_{\text {front/rear }}$ to be the average of compression and rebound damping rate

$$
d_{f / r}=\frac{d_{r e b}+d_{c o m p}}{2}
$$

the compression $d_{\text {comp }}$ and rebound damping rates $d_{r e b}$ can be determined:

$$
\begin{gathered}
d_{\text {comp }}=\frac{2 d}{1+k_{r e b, \text { comp }}} \\
d_{r e b}=\frac{2 d \cdot k_{r e b, c o m p}}{1+k_{r e b, c o m p}}
\end{gathered}
$$

\subsection{Roll Center and Anti-Roll Bar Stiffness}

The design of anti-roll bars has two objectives with respect to driving dynamics. On the one hand, the roll stiffness from the anti-roll bars is added to the existing roll stiffness of the body springs, to achieve the desired roll angle gradient. On the other hand, the distribution of the roll torque between the front and rear axle offers the potential to significantly influence the self-steer behavior of the vehicle. The distribution is designed to reduce the front axle's cornering stiffness relative to the rear axle so that ultimate traction failure under lateral acceleration first occurs in the front, causing understeer and therefore ensuring a safe driving behavior.

This effect has noticeable impact on the steering effort only at higher lateral accelerations $(>0.4 \mathrm{~g})$ due to the degression of tires [31, p.864], so that an interference with the parameterization of suspension kinematics, which is assigned to determine the steering effort at lower lateral accelerations, can be neglected. This allows a well-defined separation between the parameterization for the steering effort and driving safety, which makes this approach a clearly structured method.

Since the algorithm defines the ratio between front and rear anti-roll bar stiffness, this already takes on the role of tuning the vehicle's behavior at higher lateral acceleration in a well-defined manner. Thus, it is not intended to additionally influence the vehicle's selfsteer characteristics by altering the height of the roll centers. Referring to a comparison of common values and the suggestion of $[32, \mathrm{p} .26]$ for a roll axis slope of $0^{\circ}$ (between front and rear axle) on state-of-the-art vehicles, both roll centers are set to a neutral, generic value of $90 \mathrm{~mm}$. 
The suspension system of the vehicle may be assumed to be symmetrical with respect to the left and right sides of the vehicle. Furthermore, the front and rear roll stiffness is assumed to contribute as parallel stiffness, so that one quarter of the vehicle can serve as physical substitute model. To obtain the favored roll-angle gradient, the implemented antiroll bar stiffness is calculated as presented by Vilela et Barbosa [33]. The roll moment $M_{x}$ depends on the mass $m$, the lateral acceleration $a_{y}$ and the Center of Gravity (CG) height $h_{C G}$ :

$$
M_{x}=a_{y} \cdot m \cdot h_{C G}=a_{y} \cdot m \cdot\left(h_{R C}+h_{C G-R C}\right)=M_{x, \text { mount }}+M_{x, \text { susp }}
$$

The support of the roll moment is divided into two parts. They are distributed in accordance to the ratio between the distances from the ground to the roll axis height $h_{R C}$ on the one hand and from the roll axis height to the CG height $h_{C G-R C}$ on the other hand. Thereof, the first mentioned moment $M_{x, \text { mount }}$ is directly supported by the mountings of the wheel carrier links, while the remaining share $M_{x, s u s p}$ is supported by the suspension (springs, dampers and anti-roll bars). As the roll center positions are assumed to be fixed, both moments stay proportional to each other:

$$
\frac{M_{x, \text { mount }}}{M_{x, \text { susp }}}=\frac{a_{y} \cdot m \cdot h_{R C}}{a_{y} \cdot m \cdot h_{C G-R C}}=\frac{h_{R C}}{h_{C G-R C}}
$$

$M_{x, \text { mount }}$ is transferred only to the tire and not to the suspension springs. Therefore, the tire deflection must consider vertical forces from both moments. By multiplying the actual stiffness of the tire with $\left(1+\frac{h_{R C}}{h_{C G-R C}}\right)^{-1}$, the additional vertical force supported by the mountings is considered, too. The reduced radial tire roll stiffness $c_{\text {roll,tire,red }}$ includes the track width $s$ and the vertical stiffness of the tire $c_{\text {tire }}$. Reduced in that context means, that the vertical stiffness is transferred into roll stiffness.

$$
c_{\text {roll,tire,red }}=\frac{1}{2} \cdot c_{\text {tire }} \cdot s^{2} \cdot\left(1+\frac{h_{R C}}{h_{C G-R C}}\right)^{-1}
$$

The roll stiffness of one axle's suspension can be calculated by adding up the contribution of the springs and the anti-roll bar:

$$
c_{\text {roll,suspension }}=\frac{1}{2} \cdot c_{\text {spring }} \cdot s^{2}+\frac{1}{2} \cdot c_{\text {arb }} \cdot s^{2}
$$

To calculate the total roll stiffness of one axle, it is necessary to add up not the stiffness but the compliance of tire and suspension, since both are connected in series:

$$
c_{\text {roll,total,front/rear }}=\left(c_{\text {roll,suspension }}{ }^{-1}+c_{\text {roll,tire,red }}{ }^{-1}\right)^{-1}
$$

Now the moment equilibrium around the roll axis of the vehicle with total stiffness from both axles can be stated:

$$
a_{y} \cdot m \cdot h_{C G-R C}+M_{g}=\varphi \cdot\left(c_{\text {roll,total,front }}+c_{\text {roll,total,rear }}\right)
$$

$\mathrm{M}_{\mathrm{g}}$ represents an additional roll moment, which is caused by gravity, when the CG moves in lateral direction due to roll motion. Therefore, the center of gravity lateral position $C G_{y}$ of the vehicle has a slight offset when body roll occurs. It can be modelled by a negative stiffness since the offset depends on body roll and turns negative when shifted to the right side of equation (21):

$$
\begin{gathered}
M_{g}=\varphi \cdot m \cdot g \cdot C G_{y} \rightarrow c_{\text {gravity }}=m \cdot g \cdot C G_{y} \\
a_{y} \cdot m \cdot h_{C G-R C}=\varphi \cdot\left(c_{\text {roll,total,front }}+c_{\text {roll,total,rear }}-c_{\text {gravity }}\right)
\end{gathered}
$$


Lateral acceleration and roll angle are substituted by the desired roll-angle-gradient $\frac{\delta \varphi}{\delta a_{y}}$. The generic value for $\frac{\delta \varphi}{\delta a_{y}}$ is set to $0.45 \mathrm{~s}^{2} / \mathrm{m}$.

$$
m \cdot h_{C G-R C}=\frac{\delta \varphi}{\delta a_{y}} \cdot\left(c_{\text {roll,total,front }}+c_{\text {roll,total,rear }}-c_{\text {gravity }}\right)
$$

The ratio between the anti-roll bar stiffness in the front and rear $R_{\text {arb }}$ is empirically chosen in dependence on the longitudinal position of the CG and the overall mass of the vehicle. The effect of this ratio on self-steer behavior also depends on the roll-anglegradient, as the roll-angle-gradient determines how much the roll stiffness distribution is affected by the anti-roll bars compared to the springs. The constant values in (25) and (26) must therefore be determined empirically for the given roll-angle gradient and must be adapted manually for different values of roll-angle gradient. $R_{f}$ represents the static weight distribution to the front axle.

$$
\begin{gathered}
\text { for } R_{f}<0.5: \quad R_{a r b, r}=4+50 \cdot\left(0.5-R_{f}\right) \\
\text { for } R_{f}>0.5: \quad R_{a r b, r}=4+30 \cdot\left(0.5-R_{f}\right) \\
R_{a r b, m}=1+\frac{m-1500 \mathrm{~kg}}{8000 \mathrm{~kg}} \\
R_{a r b}=R_{a r b, r} \cdot R_{a r b, m} \stackrel{!}{=} \frac{c_{a r b, f} \cdot s_{f}^{2}}{c_{a r b, r} \cdot s_{r}^{2}}
\end{gathered}
$$

The constant value of 4 in (25) results from the requirement that an equally balanced vehicle with $R_{f}=0.5$ should have a five percent lower total stiffness (including springs and anti-roll bars) at the front axle for safety reasons than at the rear axle. The linear value of 30 results from the above requirement combined with the statement that a vehicle with $R_{f}=0.6$ does not require any further weakening of the front axle and therefore $R_{a r b, r}$ can be set to 1 . For $R_{f}<0.5$, the linear coefficient is increased from 30 to 50 for safety reasons. The calculation of $R_{a r b, m}$ was designed in a way that the first requirement is met for all vehicles regardless of the total mass.

The rear anti-roll bar stiffness can now be expressed by $R_{a r b}$ and the front anti-roll bar stiffness. As a result, the front anti-roll bar stiffness remains the only unknown quantity in (24). The obtained solution for the stiffness values satisfies both, qualitative (distribution of roll moment between front and rear) and quantitative (roll-angle-gradient) requirements.

\subsection{Suspension Kinematics}

Suspension kinematics have a large influence on the self-steer behavior of a vehicle. The kinematics determine how toe and camber angles change with vertical wheel travel and steering. The characteristics depend on the hardpoints of the suspension system. As these points need to be defined in the concept phase they are not subject to fine-tuning during the later phases of vehicle development process as this would cause high costs. To take into account these kinematic adaptions during early design phase in the parameter adaption, this subchapter shows an approach how this process can be automated.

In contrast to kinematics, suspension compliance describes the change of toe and camber due to longitudinal and lateral forces applied to the wheels. The compliance characteristics are (besides of geometric configuration of the levers) mainly determined by the stiffness of the bushings. Even though compliance behavior is also targeted in the concept phase, the stiffness of the bushings can be modified in later phases of development, while the position of the hardpoints is fixed then.

Therefore, only kinematics are considered for the parameter adaption, in particular the change of toe and camber. In the following, all kinematic characteristics are approximated 
using the linear equation (28). The corresponding angle gamma (toe or camber) is expressed in dependence on the variation of wheel travel. Positive $z_{\text {wheel }}$ means compression. $\gamma$ is positive for positive camber (top of the wheel moves out) and positive for positive toe (front of the wheel moves in).

$$
\gamma=p_{1} \cdot z_{\text {wheel }}+p_{0}
$$

The camber kinematics partially compensate the vehicle's roll angle to ensure that the tire-road contact not only is located on the tire's outer flank or even on the shoulder. When comparing common values for camber from a summary of different cars' suspension setups in [32, p.32-33] and [34], it can be concluded that the static $\left(\mathrm{p}_{0}\right)$ and kinematic values $\left(\mathrm{p}_{1}\right)$ at the rear axle are chosen to be approximately $50 \%$ to $100 \%$ more negative than at the front axle because of the lateral force potential of the rear wheels being crucial for driving stability. Furthermore, excessive camber changes during wheel travel are generally to be avoided for reasons of straight-line stability [32, p.34]. Taking these guidelines into account, the following generic values in Table 2 are chosen:

Table 2. Generic static and kinematic camber values for a state-of-the-art vehicle.

\begin{tabular}{|l|l|l|l|}
\hline & & $\mathrm{p}_{1}$ & $\mathrm{p}_{0}$ \\
\hline & Camber front & $-10^{\circ} / \mathrm{m}$ & $-0.5^{\circ}$ \\
\hline & Camber rear & $-16^{\circ} / \mathrm{m}$ & $-1^{\circ}$ \\
\hline
\end{tabular}

As already mentioned, the influence of the camber values is to be kept at a constant level for reasons of comparability. Purely constant coefficients can only be used for the static value $\mathrm{p}_{0}$. The vertical wheel travel depends on the roll stiffness and the track width. Therefore, the $p_{1}$ values given in Table 2 must be adapted, if a vehicle's track width differs from the reference track width of $1.7 \mathrm{~m}$.

Since the maximum lateral force of a tire is not primarily affected by in- or decreasing the steering angle of a wheel (in contrast to altering the camber), it is possible to influence the self-steer behavior of the vehicle using the toe kinematics without compromising the general vehicle's lateral potential, thus not risking a decrease in driving safety. The focus of designing the toe kinematics is to achieve a neutral, comparable behavior regarding steering effort in the linear region of lateral dynamics for any powertrain configuration and therefore every different set of chassis parameters.

The specific aim of this parameterization is to analytically calculate how to achieve the required additional steering angle via toe kinematics when cornering to reach the same steering effort as a nearly neutral reference vehicle. This demand yields to equation (24), when $\mathrm{k}_{\mathrm{u} 1}$ is considered the reference understeer gradient and $\mathrm{k}_{\mathrm{u} 2}$ the understeer gradient of the vehicle, which is subject to parametrization. $\delta_{\mathrm{A}}$ corresponds to the slipless cornering Ackermann steering angle.

$$
\delta_{1} \stackrel{!}{=} \delta_{2}-\delta_{\text {Kinematic }} \quad \rightarrow \quad \delta_{A}+k_{u 1} \cdot a_{y} \stackrel{!}{=} \delta_{A}+k_{u 2} \cdot a_{y}-k_{\text {kinematic }} \cdot a_{y}
$$

Equation (24) indicates that there is the need for an additional steering angle of being dependent on the lateral acceleration of the vehicle. This requirement is met since the body's roll angle is assumed to be linearly dependent on lateral acceleration (23). Equation (30) describes how the kinematic steering angle is calculated with respect to body roll (wheel travel due to roll motion $z_{\text {Roll }}$ ) and the toe coefficient $\mathrm{p}_{1}$ (not the camber coefficient from Table 2).

$$
\delta_{\text {Kinematic }}=k_{\text {kinematic }} \cdot a_{y}=p_{1} \cdot z_{\text {Roll }}=p_{1} \cdot s \cdot \varphi
$$

The body roll angle is given by equation (31), where $c_{\text {roll,total,front/rear }}$ (referring to the explanation of anti-roll stiffness in subsection 3.6) is the total resistance against roll.

$$
\varphi=\frac{M_{x, \text { susp }}}{c_{\text {roll,total,front/rear }}}=\frac{h_{C G-R C} \cdot m \cdot a_{y}}{c_{\text {roll,total,front } / \text { rear }}}
$$


When combining (30) with (31) and inserting the results into (29), it is possible to formulate correlation (32) between the configuration's self-steer characteristics and the needed coefficient for toe kinematics:

$$
\delta_{A}+k_{u 1} \cdot a_{y}=\delta_{A}+k_{u 2} \cdot a_{y}-p_{1} \cdot s \cdot \frac{h_{C G-R C} \cdot m}{c_{\text {roll,total,front } / \text { rear }}} \cdot a_{y}
$$

The unknown coefficient $\mathrm{p}_{1}$ is finally obtaining from equation (32)

$$
p_{1}=\left(k_{u 2}-k_{u 1}\right) \cdot \frac{c_{\text {roll,total,front } / \text { rear }}}{s \cdot h_{C G-R C} \cdot m}
$$

The reference understeer gradient $\mathrm{K}_{\mathrm{u} 1}$ is a freely selectable parameter and can be chosen to match the desired steering effort. For a neutral and agile reference with a slight tendency to understeer, thus meeting safety requirements, the specific value for this study is set to $\mathrm{k}_{\mathrm{u} 1}$ $=0.000325 \mathrm{~s}^{2} / \mathrm{m}$.

Since the complete kinematics parameterization is expected to be run analytically, the understeer gradient of the current configuration $\mathrm{k}_{\mathrm{u} 2}$ is calculated using a single-track model (34), which eliminates the need for time-consuming iterative simulation.

$$
k_{u 2}=\frac{m}{l} \cdot\left(\frac{l_{r}}{c_{f, \text { tire }} \cdot k_{S}}-\frac{l_{f}}{c_{r, \text { tire }}}\right)
$$

The values for front and rear tire cornering stiffness $\left(c_{f, \text { tire }}\right.$ and $\left.c_{r, \text { tire }}\right)$ are obtained in dependence on the vertical wheel load from the Pacejka tire model used in simulation. $\mathrm{k}_{\mathrm{s}}$ was determined to be 0.94 to include the influence of the anti-roll bar, spring stiffness distribution and camber values in the single-track model.

\section{Results}

To evaluate the results, a parameter variation is performed. The baseline configuration of the glider is set to the specifications of a Tesla Model S 85D (main Data listed in Table 3). The powertrain definition and the tire model (including the rolling resistance) were also specified accordingly.

Table 3. Main input parameters for the glider.

\begin{tabular}{|l|l|l|}
\hline & Quantity & Value \\
\hline & Wheelbase & $2960 \mathrm{~mm}$ \\
\hline & Trackwidth front & $1662 \mathrm{~mm}$ \\
\hline & Trackwidth rear & $1700 \mathrm{~mm}$ \\
\hline & Total width & $1964 \mathrm{~mm}$ \\
\hline & Total length & $4978 \mathrm{~mm}$ \\
\hline & Air drag coefficient & 0.24 \\
\hline & Frontal area & 2.34 \\
\hline & Wheel dimensions & $245 / 45 \mathrm{R} 19$ \\
\hline & Rolling resistance & 0.0086 \\
\hline & Range (NEDC) & $502 \mathrm{~km}$ \\
\hline
\end{tabular}

For this baseline vehicle, the longitudinal weight distribution as well as the total weight have been modified. The equal weight distribution of the base vehicle was adapted to $60 \%$ front $/ 40 \%$ rear and vice versa while retaining the total weight. By that, the total range for any configuration is covered, as no realistic concept with an underfloor battery is likely to have a weight distribution that deviates more than $10 \%$ from a equal distribution. Additionally, the total weight has been decreased and increased about $10 \%$ while keeping the load distribution constant. A total weight change of $+/-10 \%$ again represents the maximal weight change due to powertrain design modifications. 


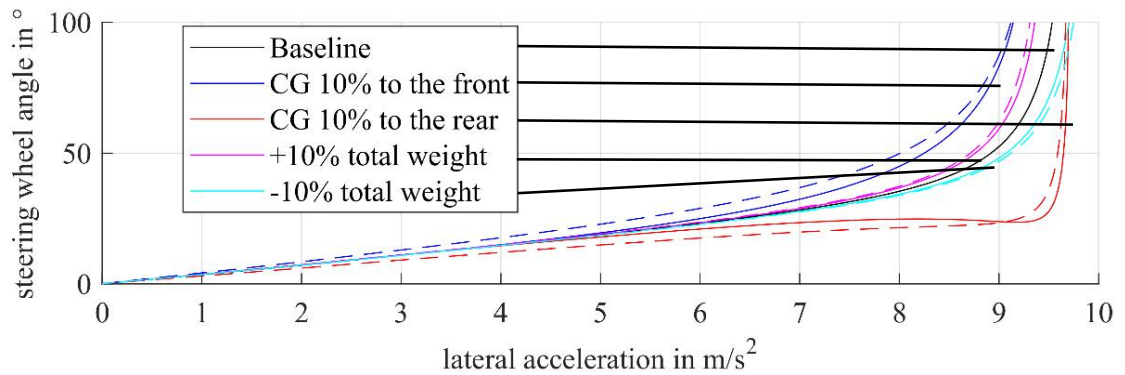

Fig. 3. Plausibilisation of steering behavior for different configurations (solid:adaptive, dashed: nonadaptive).

The comparison of the steady-state cornering behavior of these vehicles is shown in Figure 3. In the linear region up to $5 \mathrm{~m} / \mathrm{s}^{2}$, different configurations match well when using the parameter adaption. Without adaptive parameters, the configurations with a modified weight distribution deviate from the target behavior, while changes in weight do not affect the behavior. In the nonlinear region, the effect of the adaptive parameters is minor in comparison to the effect caused by the variations. The weight distribution again shows a higher influence than the total weight does. The configuration with the CG shifted to rear still shows an understeering behavior in the near-limit area. This is caused by the stiffness of the front axle that makes the front tires reach their degression first.

The transient behavior is evaluated with Bode plots of the yaw rate in a sine sweep steering maneuver. Results are shown in Figure 4. For low frequencies, the results for adaptive parameters are consistent with the steady state results, while the deviations increase with frequencies higher than $0.8 \mathrm{~Hz}$. Again, the modifications in the total weight cause minor effects compared to the influences caused by a modified CG position. The simulation results for non-adaptive sets of suspension parameters deviate when modifying the weight distribution. This is a result from the deviations in the steady-state cornering behavior from Figure 3.
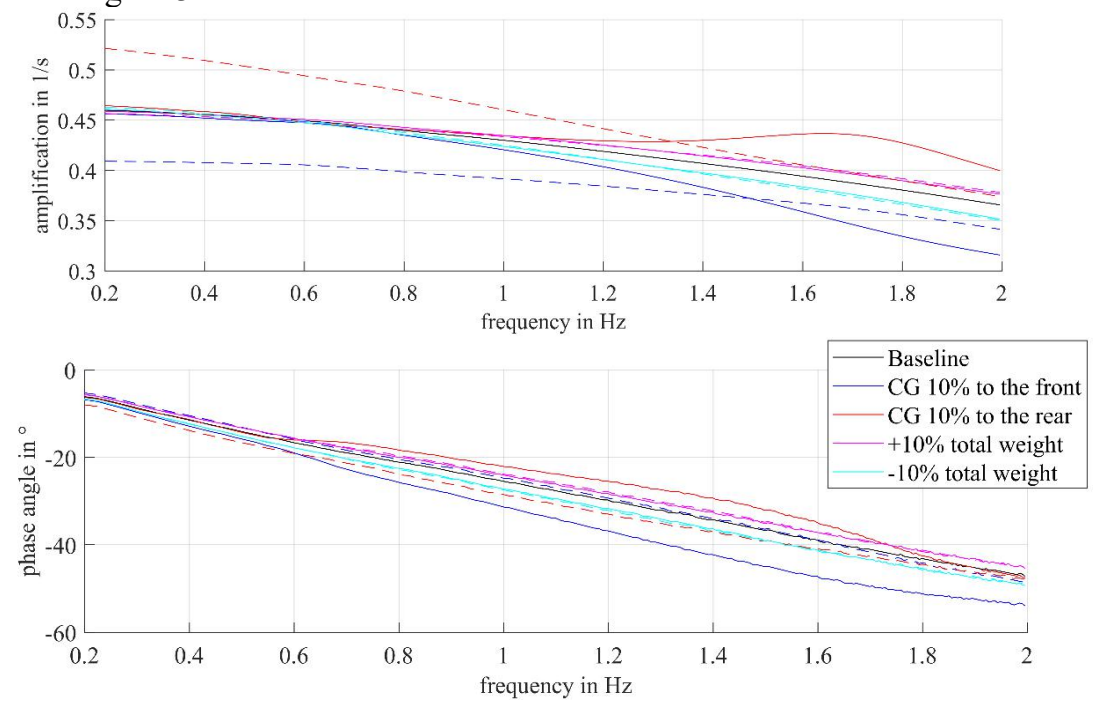

Fig. 4. Bode plot for the steering angle of different configurations during in sine sweep steering manouver (solid:adaptive, dashed: non-adaptive.

\section{Summary and Discussion}


The literature review showed a need for the consideration of vehicle dynamics in powertrain design optimization for multi-motor EV. It was further showed that such a holistic powertrain design optimization has not been conducted yet. The authors then describe the methodology for the adaptive parameterization. In particular, they explain in detail the determination of parameters for springs, dampers, anti-roll-bars as well as suspension kinematics. Results prove that modifications in the total weight and the weight distribution due to changes in the powertrain configuration have major effects on the vehicle's dynamic behavior. The presented method for an automated parameter adaption for the suspension design is able to align the steady-state behavior in the linear region. The non-linear region can only be influenced in a minor way. The difference between the adaptive and the non-adaptive sets of parameters in steady-state conditions in general is quite low. In general, the adaptive parameters have large effects on the overall evaluation of a powertrain concept, in particular due to changes in total weight. Thus, it is necessary to ensure the comparability of different powertrain layouts.

While the approach can be applied to many types of vehicles, an adaption of certain constant values might be necessary (roll center height, Eigen frequency, damping ratio) due to different requirements. Furthermore, the approach cannot ensure an optimal behavior of different configurations regarding vehicle dynamics, as this would require a separate optimization. However, a separate optimization of the suspension design within a powertrain design optimization would lead to extensive and inacceptable computational efforts. Furthermore, it would to be too detailed for the early design phase. Therefore, this approach can be seen as a trade-off solution between low computational effort and comparability of results.

The vehicle behavior most likely is influenced by changes in the CG position. The range of variation shown above from $40 / 60$ to $60 / 40$ can be seen as a limit for most vehicle concepts. By intelligent positioning of components in the vehicle package (in particular the battery), a 50/50 load distribution can be achieved in most cases for electric vehicles. The change in total weight is more likely to appear for different powertrain configurations due to different efficiencies as well as power demands and the subsequent adaption of the battery to maintain the necessary maximum power and energy.

\section{Conclusion and Outlook}

The presented method improves the comparability of vehicle dynamics simulations in the context of powertrain design optimization. Nevertheless, the effect is not too large compared to a non-adaptive choice of parameters. Changes in the CG position affect the results more than a change in the total weight.

Future research will focus on powertrain design optimization with consideration of vehicle dynamics. The adaptive parameterization will be included in a powertrain design optimization to ensure a valid suspension design as well as accurate weight and inertia parameters. One thing that is not included in the parameter adaption is an adaption of the tire dimensions. [35] proposes a method to adaptively scale tire parameter sets for Pacejka tire models. In future research, an integration of tire scaling will be evaluated.

\section{Acknowledgments}

Thanks to Andreas Schultze for his ideas and hints in several discussions. We also thank Lorenzo di Caro, Stefano Gabriele, Tobias Zuchtriegel and Andreas Holtz, who also worked on vehicle dynamics topics in the project during their scientific thesis work. The research was conducted with basic research funds from the Institute of Automotive Technology, Technical University of Munich. Thanks to our colleagues from the Center for 
Design Research at Stanford, where part of the work was done during a 3 month research visit.

\section{Contributions}

As first author, Christian Angerer defined the approach and mainly developed the presented method. His contribution was approximately $75 \%$. Benedikt Mößner contributed main parts to the parameter adaption, in particular the weight calculation and the suspension design as part of his semestral thesis. Moritz Lüst revised the anti-roll bar design and added the kinematics part in his semestral thesis. He furthermore contributed the formulation of equations to this paper. Stefan Büchner contributed major knowledge regarding the design of suspension kinematics and compliance. Markus Lienkamp made an essential contribution to the conception of the research project. He revised the paper critically for important intellectual content. Felix Sträußl contributed additional ideas during discussions on the final paper. Markus Lienkamp gave final approval of the version to be published and agrees to all aspects of the work. As a guarantor, he accepts responsibility for the overall integrity of the paper.

\section{References}

1. A. Pennycott, L. de Novellis, A. Sabbatini, P. Gruber, and A. Sorniotti, "Reducing the motor power losses of a four-wheel drive, fully electric vehicle via wheel torque allocation," Proceedings of the Institution of Mechanical Engineers, Part D: Journal of Automobile Engineering, vol. 228, no. 7, pp. 830-839, 2014.

2. C. Angerer, S. Krapf, A. Buß, and M. Lienkamp, "Holistic modeling and optimization of electric vehicle powertrains considering longitudinal performance, vehicle dynamics, costs and energy consumption: (accepted)," in Technical Conferences and Computers and Information in Engineering Conference: Proceedings, Quebec, Canada, 2018.

3. C. Angerer, M. Felgenhauer, I. Eroglu, M. Zähringer, and M. Lienkamp, "Scalable Dimension-, Weight- and Cost-Modeling for Components of Electric Vehicle Powertrains: (submitted)," in 21st International Conference on Intelligent Transportation Systems: Proceedings, Maui, 2018.

4. L. de Novellis, A. Sorniotti, and P. Gruber, "Design and comparison of the handling performance of different electric vehicle layouts," Proceedings of the Institution of Mechanical Engineers, Part D: Journal of Automobile Engineering, vol. 228, no. 2, pp. 218-232, 2013.

5. H. Wu and H. Zhang, "Model-Based Design and Evaluation of Electric Vehicle Powertrain With Independent Driving Motors," in International Design Engineering Technical Conferences and Computers and Information in Engineering Conference: Proceedings, Boston, Massachusetts, USA, 2016.

6. Z. Ye, M. F. Stapelbroek, J.-S. R. Pfluger, T. Reckeweg, and R. Savelsberg, "Smart Torque Vectoring Functionality for AWD Electric Vehicles," in 30th International Electric Vehicle Symposium \& Exhibition: Proceedings, Stuttgart, 2017.

7. M. Greger, "Auswirkung einer variablen Momentenverteilung auf die Fahrdynamik," Dissertation, Institute of Automotive Technology, Technical University of Munich, Munich, 2006.

8. L. de Novellis, A. Sorniotti, P. Gruber, L. Shead, V. Ivanov, and K. Hoepping, "Torque vectoring for electric vehicles with individually controlled motors: state-of-the-art and 
future developments," in 26th International Electric Vehicle Symposium, Los Angeles, 2012.

9. M. Papageorgiou, M. Leibold, and M. Buss, Optimierung. Berlin, Heidelberg: Springer Berlin Heidelberg, 2012.

10. U. Diwekar, Introduction to Applied Optimization. Boston, MA: Springer US, 2008.

11. K. Deb, A. Pratap, S. Agarwal, and T. Meyarivan, "A fast and elitist multiobjective genetic algorithm: NSGA-II," IEEE Transactions on Evolutionary Computation, vol. 6, no. 2, pp. 182-197, 2002.

12. K.-W. Jeon, T.-K. Chung, and S.-C. Hahn, "NEMA class a slot shape optimization of induction motor for electric vehicle using response surface method," in International Conference on Electrical Machines and Systems (ICEMS): Proceedings, Beijing, China, 2011, pp. 1-4.

13. I. Eroglu, L. Horlbeck, M. Lienkamp, and C. M. Hackl, "Increasing the overall efficiency of induction motors for BEV by using the overload potential through downsizing," in International Conf. on Electric Machines and Drives Conference (IEMDC): Proceedings, Miami, FL, USA, 2017, pp. 1-7.

14. P. K. Choudhary, S. P. Dubey, B. Tiwari, and B. Dewangan, "Efficiency optimization of induction motor drive using Artificial Neural Network," in Energy Efficient Technologies for Sustainability (ICEETS), 2016 International Conference on, Nagercoil, India, 2016, pp. 821-827.

15. N. M. Patel, B.-S. Kang, J. E. Renaud, and A. Tovar, "Crashworthiness Design Using Topology Optimization,” J. Mech. Des., vol. 131, no. 6, p. 61013, 2009.

16. Z. Zhou, K. Hamza, and K. Saitou, "Decomposition Templates and Joint Morphing Operators for Genetic Algorithm Optimization of Multicomponent Structural Topology," J. Mech. Des., vol. 136, no. 2, p. 21004, 2014.

17. G. Domingues, A. Reinap, and M. Alakula, "Design and cost optimization of electrified automotive powertrain," in International Conference on Electrical Systems for Aircraft, Railway, Ship Propulsion and Road Vehicles \& International Transportation Electrification Conference (ESARS-ITEC), Toulouse, France, 2016, pp. $1-6$.

18. [18] M. J. Alexander, J. T. Allison, and P. Y. Papalambros, "Decomposition-based design optimisation of electric vehicle powertrains using proper orthogonal decomposition," IJPT, vol. 1, no. 1, p. 72, 2011.

19. M. Fries, S. Wolff, L. Horlbeck, M. Kerler, M. Lienkamp, A. Burke, and L. Fulton, "Optimization of hybrid electric drive system components in long-haul vehicles for the evaluation of customer requirements," in 12th International Conference on Power Electronics and Drive Systems (PEDS): Proceedings, Honolulu, HI, 2017, 1,141-1,146.

20. A. Schönknecht, A. Babik, and V. Rill, "Electric Powertrain System Design of BEV and HEV Applying a Multi Objective Optimization Methodology," Transportation Research Procedia, vol. 14, pp. 3611-3620, 2016.

21. A. E. Baumal, J. J. McPhee, and P. H. Calamai, "Application of genetic algorithms to the design optimization of an active vehicle suspension system," Computer Methods in Applied Mechanics and Engineering, vol. 163, no. 1-4, pp. 87-94, 1998.

22. J. P. C. Gonalves and J. A. C. Ambrsio, "Road Vehicle Modeling Requirements for Optimization of Ride and Handling," Multibody Syst Dyn, vol. 13, no. 1, pp. 3-23, 2005. 
23. A. C. Mitra, G. J. Desai, S. R. Patwardhan, P. H. Shirke, W. M.H. Kurne, and N. Banerjee, "Optimization of Passive Vehicle Suspension System by Genetic Algorithm," Procedia Engineering, vol. 144, pp. 1158-1166, 2016.

24. J. Tobolar, R. Castro, de, U. Bleck, C. Satzger, and J. Brembeck, "Comparative evaluation of energy efficiency of electrical vehicle powertrain configurations," in 24th Symposium of the International Association for Vehicle System Dynamics (IAVSD 2015), Graz, 2015.

25. A. Schultze and M. Lienkamp, "Potential of an improved energy efficiency in the chassis," Automot. Engine Technol., vol. 1, no. 1-4, pp. 15-25, 2016.

26. S. Fuchs, "Verfahren zur parameterbasierten Gewichtsabschätzung neuer Fahrzeugkonzepte," Dissertation, Institute of Automotive Technology, Technical University of Munich, Munich, 2014.

27. D. D. MacInnis, W. E. Cliff, and K. W. Ising, "A Comparison of Moment of Inertia Estimation Techniques for Vehicle Dynamics Simulation," SAE Technical Paper, no. 970951, 1997.

28. M. Abe, Ed., The dynamics of vehicles on roads and on tracks: Proceedings of the 18th IAVSD symposium held in Kanagawa, Japan, August 24 - 30, 2003. London: Taylor \& Francis, 2004.

29. M. Felgenhauer, C. Angerer, R. Marksteiner, F. Schneider, and M. Lienkamp, "Geometric substitute models for efficient scaling of dimensions during vehicle architecture design: (in press)," in Proceedings: 15th International Design Conference, Dubrovnik, 2018.

30. I. Preda, "About the preliminary design of the suspension spring and shock absorber," IOP Conf. Ser.: Mater. Sci. Eng., vol. 147, p. 12128, 2016.

31. S. Pischinger and U. Seiffert, Vieweg Handbuch Kraftfahrzeugtechnik. Wiesbaden: Springer Fachmedien Wiesbaden, 2016.

32. B. Heißing, M. Ersoy, and S. Gies, Fahrwerkhandbuch. Wiesbaden: Springer Fachmedien Wiesbaden, 2013.

33. D. Vilela and R. S. Barbosa, "Analytical models correlation for vehicle dynamic handling properties," J. Braz. Soc. Mech. Sci. \& Eng., vol. 33, no. 4, pp. 437-444, 2011.

34. M. Mitschke and H. Wallentowitz, Dynamik der Kraftfahrzeuge. Wiesbaden: Springer Fachmedien Wiesbaden, 2014.

35. A. Schultze, "Energieeinsparpotential im Fahrwerk von Kraftfahrzeugen," Dissertation (submitted), Lehrstuhl für Fahrzeugtechnik, Technical University of Munich, Munich, 2018. 